\title{
Factors affecting reports of fragmenting visual images*
}

\author{
JOHN R. SCHUCK \\ Bowling Green State University, Bowling Green, Ohio 43403
}

\begin{abstract}
Seven experiments employing simulation as well as actual voluntary stabilization were performed to study the reporting of fragmenting visual images. The results indicated a significant bias toward the reporting of meaningful fragments when verbal reports were used throughout each trial. Moreover, tracings of fragments were completely accurate only when one line segment of the original pattern remained, with errors becoming increasingly likely as the number of segments remaining increased. Diagonally oriented line segments disappeared more frequently than those oriented either vertically or horizontally. Locus of fixation proved important, but Hebb's hypothesis concerning the preponderance of meaningful fragments was not supported.
\end{abstract}

When an optical device, such as a contact lens bearing a miniature projector, is used to stabilize a pattern on the retina, the viewer typically reports either its fragmentation or total disappearance within seconds. This phenomenon was used by Pritchard, Heron, and Hebb (1960) to study the fragmentation of a variety of visual figures, among them an " $B$ " pattern. An analysis of the Ss' verbal reports led the investigators to conclude that fragmentation was not random, since, when any part of the " $B$ " figure remained, it always contained one or more of the meaningful, complete symbols " 4 ," "B," or "3." Hebb (1963) used these observations to support his theoretical views concerning the influence of past experience upon perceptual organization. Unfortunately, the data from which Hebb's conclusions were drawn remain equivocal in view of at least two methodological flaws in Pritchard's procedure.

Since stabilization sometimes produces changes as frequently as one each second, the Ss may not have reported or remembered those fragments difficult to describe with words. Moreover, the contact lens systems are relatively costly and difficult, and so observations have typically been limited to a very few persons. Usually, the Es have simply fitted themselves with the contact lens system. This gives the E direct control over the dependent variable in his study and presents the possibility for subtle and unintentional biasing of the data.

The Ss who voluntarily fixate a pattern will, under certain conditions, report disappearances. Clark (1957, 1960, 1961) has presented considerable evidence to show that optical stabilization and the disappearances reported under conditions of voluntary inhibition of head, eye, and blinking movements are manifestations of the same underlying process. For both situations, the

*This work was supported by Grant B6-0249 from the Psychobiology Program of the Biological and Medical Sciences Division of the National Science Foundation. I want to thank Michael Doherty for his critical comments on an earlier version of the manuscript. manipulation of color, intensity, contrast, size, and retinal location result in comparable effects, differing only in degree. Forde, Piggins, and MacKinnon (1966) compared the contact lens approach with voluntary fixation and with another viewing condition in which an intense flash of light was used to create a prolonged afterimage of the visual pattern. Since these three different conditions produced the same kinds of fragmentations with comparable relative frequencies, these authors also concluded that a common perceptual process was involved.

Using voluntary fixation, McKinney's (1963) Ss reported a preponderance of meaningful fragments comparable to those described by Pritchard, Heron, and Hebb (1960). However, Schuck and Leahy (1966) found that verbal reports were strongly biased in favor of meaningful elements. Perhaps response bias rather than perceptual organization is responsible for this preponderance of meaningful fragments.

The major purpose of the experiments reported here was to obtain additional evidence about possible response biases in these kinds of perceptual reports. Fragmenting images were simulated either on moving picture film or by programming the appearance and disappearance of line segments on an electroluminescent, alphanumeric panel. These simulated fragments provided objective criteria for judging the adequacy of various reporting methods.

The Ss in all experiments were volunteers from introductory psychology courses. Unless otherwise noted, they received class credit for participating.

\section{EXPERIMENT I}

One purpose of this experiment was to provide data for later simulation of a fragmenting " $B$ " figure. Another was to determine if two different reporting methods affected the size, complexity, or meaningfulness of reported fragments. 


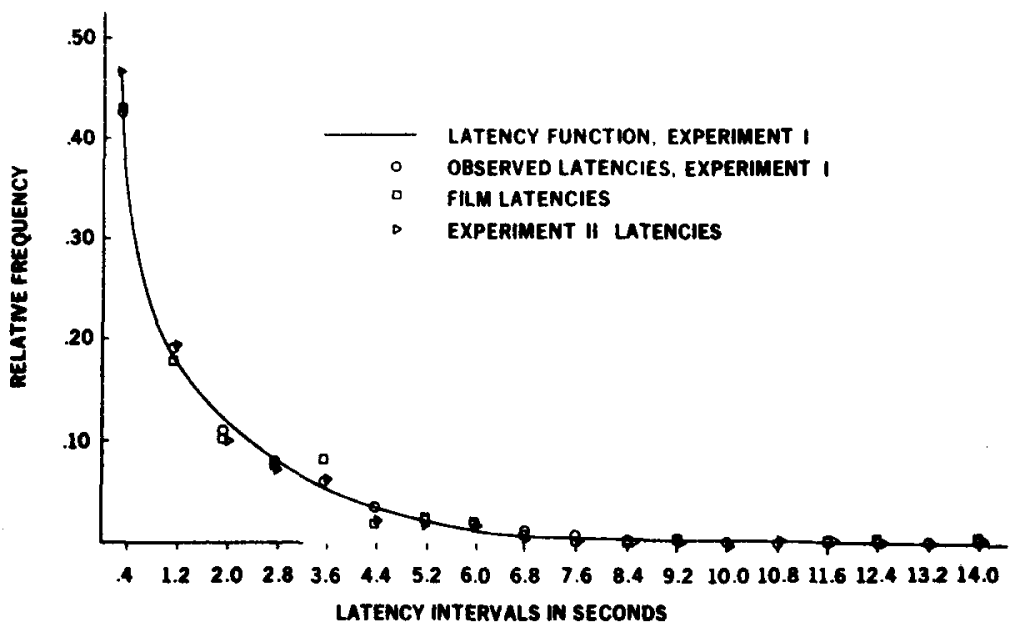

Fig. 1. A comparison of latencies from Experiments I and II. The line labeled "latency function" was fit by visual inspection to the observed data points obtained in Experiment $I$.

\section{Method}

\section{Subjects}

The Ss were 22 male and 18 female students. Eleven Ss, 5 men and 6 women, were paid for participating.

\section{Apparatus}

The $S$ sat in front of a table in a lightproof room. A luminous "H" figure was centered at approximately eye level on a flat-black plywood partition just $\mathbf{4 2}$ in. in front of $S$ s. The figure was obtained by mounting a 12-in.-square electroluminescent panel (Sylvania PM-144) on the partition, then overlaying the panel with a high-contrast photographic negative of the $\mathrm{HB}$. Overall, the figure was 7 in. high $\times 8$ in. wide and was constructed of lines $1 / 2$ in. wide. Luminance was approximately $.1 \mathrm{fL}$. When viewed in the dark, the pattern appeared greenish-gray on a dark ground.

A small brass knob, about $3 / 8$ in. in diam and $1 / 4$ in. high, was bolted through the table top just in front of $S$. This knob was connected to a contact relay so that it served as a contact switch for reporting the latencies and durations of fragments. The table top was covered with a black cloth to prevent any reflection. Solid-state programming and control modules were used to program the stimulus presentations.

\section{Procedure}

Each $S$ was instructed to fixate on the intersection of the horizontal line and the rightmost vertical line of the $\mathrm{HB}$ figure; he was told to keep his head and eyes motionless and to refrain from blinking as long as possible. The room lights were automatically turned off at the beginning of each trial as a signal for $S$ to get ready. Five seconds later, the pattern was lighted. The instructions included the following: "We want to know how of ten fragments occur and how long each one lasts. You are to report this by lightly touching the contact switch once for each fragment. Keep your finger on the switch as long as the fragment remains unchanged. If a new fragment appears, lift your finger momentarily and then touch the switch again, continuing to touch the switch for the duration of the new fragment."

For each trial, $S$ was provided with an $8 \frac{1}{2} \times 11$ in. sheet of paper, upon which a full-sized outline of the stimulus figure was printed in faint, blue lines. The $S$ was told that at some time during the trial, the room lights would go on when he touched the contact switch to report a fragment. When this happened, he was to use a felt marking pen to trace accurately and completely those portions of the pattern representing the fragment he had just seen.

The latency of a particular fragment was defined as the amount of time the visual pattern appeared as an intact whole before fragmenting. The duration of a fragment was the amount of time that the fragment remained unchanged. Latencies and durations were recorded for the first $45 \mathrm{sec}$ of each trial, and after this initial interval, the next reported fragment turned the room lights on and the trial ended with S's tracing. Half of the Ss traced those parts of the figure that disappeared; the other half traced those parts that remained. All Ss viewed the figure monocularly. The right eye was occluded for half of the Ss, the left eye for the other half. Each $S$ was given six practice trials followed by six experimental trials.

\section{Results and Discussion}

Overall, the Ss reported 3,870 fragmentations, or an average of 16 for each trial. Figures 1 and 2 show these latencies and durations in the form of probability distributions pooled across all 40 Ss. The mean latency was $1.93 \mathrm{sec}$ with a standard deviation of 2.34 , and the mean duration was $1.30 \mathrm{sec}$ with a standard deviation of 1.26 .

In addition, 240 tracings were obtained of the last fragment reported on each trial. Of these, 120 were tracings of the part that disappeared (the D set) and 120 were tracings of the part that remained (the $R$ set). In order to compare these two different ways of reporting, all tracings were analyzed with respect to the part that remained; for the remainder of this paper, the term fragment will refer to the part of the stimulus pattern remaining, regardless of whether the $\mathrm{S}$ was actually indicating the part that disappeared or the part that remained.

The $\mathrm{D}$ set of tracings was compared to the $\mathrm{R}$ set on three different indices, one of the complexity of the fragments, another of the amount of the original pattern left intact, and the third of the meaningfulness of the fragments.

Complexity was measured by the number of 
Fig. 2. A comparison of durations from Experiments I and II. The duration function was fit by visual inspection to the observed durations obtained in Experiment $I$.

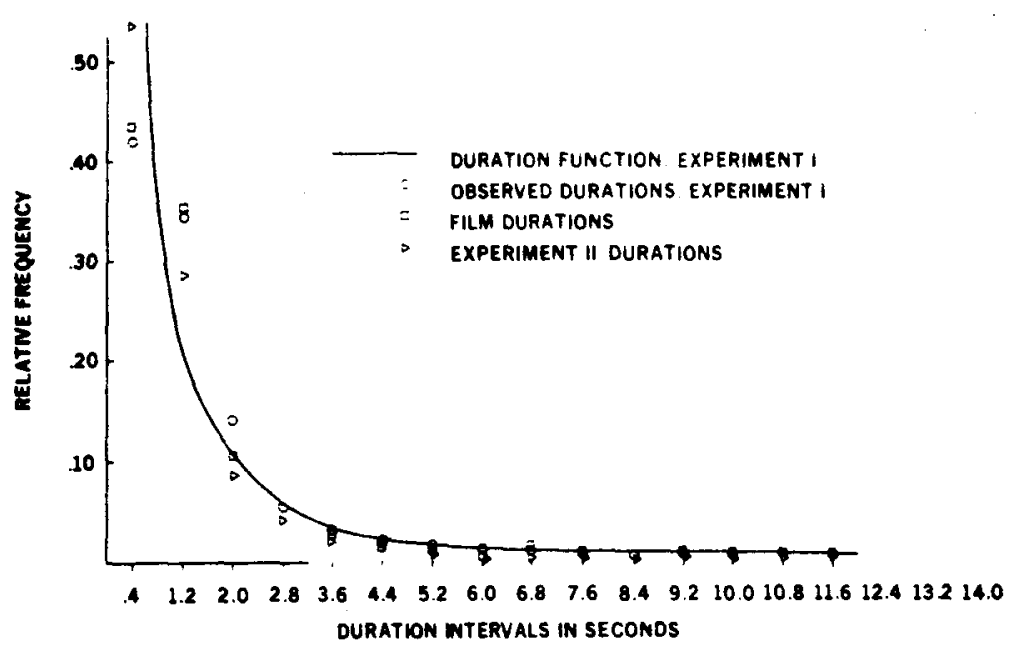

discontinuities in the fragment, when a discontinuity was defined as the number of times a person tracing the pattern would have to lift his pencil from the data sheet in order to trace accurately the fragment with retracing allowed. The number of tracings in the $\mathrm{D}$ set having zero, one, and two or more discontinuities were 63,44 , and 13, respectively; the analogous frequencies for the $R$ set were 64,61 , and 15 . Thus, the $D$ and $R$ sets were quite similar in complexity.

In order to find out if these two sets differed with respect to the amount of the original figure left intact, the linear extent of each fragment was measured. The mean linear extent was 19.4 in. for the group reporting the parts that remained (Group R) and 20.0 in. for those reporting the parts that disappeared (Group D). The difference between these means was not significant $(t=$ .37 ; $\mathrm{df}=38$ ).

In order to avoid the problem of scaling the meaningfulness of each reported fragmentation, only reports of an intact $\mathrm{H}, \mathrm{h}, \mathrm{B}, \mathrm{b}, 1 \mathrm{~B}, 1,3,11,13,113$, and + were considered. These disappearances satisfy Pritchard, Heron, and Hebb's (1960) implicit definition of meaningfulness (letters, numerals, common symbols) and can be scored objectively.

The mean proportion of fragments from this arbitrary set was .27 for Group $R$ and .18 for Group D. A t test indicated that these means were not significantly different $(t=1.21 ; \mathrm{df}=38)$.

In the complexity analysis, two judges independently classified each fragment according to the number of hypothetical perceptual elements required to produce that fragment. Figure 3a illustrates an $\mathbf{B}$ figure made up of eight straight and two curved line elements. The assumption underlying this hypothetical figure is that during fragmentation each one of the 10 segments involved either remains intact or totally disappears. This is the simplest hypothetical structure that can account for the kinds of fragments reported by Pritchard. Heron. and Hebb (1960): 4s, 3s, 13s, hs, bs, etc.

A large portion of the 240 fragments could not be accounted for by these kinds of units, however. In a number of tracings, for example, only about half of the upper curved portion of the " $B$ " remained or only about half of the horizontal line of the "H." The hypothetical structure pictured in Fig. 3b is necessary to account for these kinds of fragments. Those tracings which were too complex to be accounted for by either of these underlying structures were assigned to a third category.

In other words, the judges independently assigned each reported fragment to one of three categories according to whether the fragment required 10 or less, from 11 to 20 , or more than 20 hypothetical perceptual units comprising the $\mathbf{H}$ figure. If these categories are referred to as $\mathrm{A}, \mathrm{B}$, and $\mathrm{C}$, respectively, then Judge 1 classified $42 \%$ of the fragments in Category A, 23\% in B, and $35 \%$ in $\mathrm{C}$. For Judge 2, the respective figures were $39 \%, 28 \%$, and $33 \%$.

An informational analysis was used to describe the extent of agreement among the judges (Garner, 1962). The uncertainty associated with the three classification categories was 1.558 bits, while the conditional uncertainty of classification categories given judges was 1.556. Hence, more than $99 \%$ of the information (or loosely, variance) in classification was still accounted for

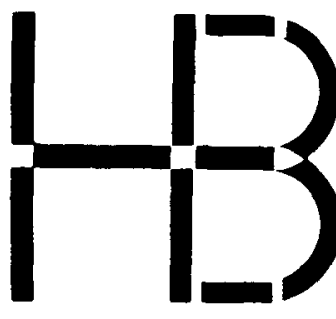

a

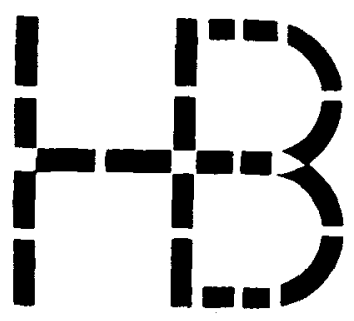

b
Fig. 3. Two of the hypothetical perceptual structures used to categorize the complexity of fragments reported in Experiment I. 
Table 1

Summary Statistics for the Distributions of Latencies and Durations in the Original HB Data, the Simulation Film, and Experiment II

\begin{tabular}{|c|c|c|c|}
\hline & $\begin{array}{l}\text { Mean } \\
(\mathrm{Sec})\end{array}$ & $\begin{array}{c}95, c \text { Confidence } \\
\text { Interval About } \\
\text { the Mean }\end{array}$ & $\mathrm{SD}$ \\
\hline \multicolumn{4}{|l|}{ Latencies } \\
\hline Original Observed & 1.93 & & 2.34 \\
\hline Simulation Film & 2.01 & $1.74-2.28$ & 2.41 \\
\hline Experiment II & 1.78 & $.93-2.63$ & 2.20 \\
\hline \multicolumn{4}{|l|}{ Durations } \\
\hline Original Observed & 1.30 & & 1.26 \\
\hline Simulation Film & 1.41 & $1.25-1.57$ & 1.70 \\
\hline Experiment II & 1.19 & $.59-1.79$ & 1.61 \\
\hline
\end{tabular}

when variation due to judges was statistically removed.

In summary, whether $S$ reported the part that disappeared or the part that remained had no significant effect on the complexity, extent, or the meaningfulness of his tracings. Moreover, these tracings were not predominantly of simple, meaningful forms.

\section{EXPERIMENT II: A COMPARISON OF FOUR DIFFERENT KINDS OF REPORTS}

This study was designed to evaluate four different methods of reporting fragments. Since reporting methods were the major concern, not actual fragmentation, Ss reported a filmed simulation of the phenomenon. In this way, the proportion of meaningful fragments in Ss' reports could be compared with the actual proportion in the film.

One specific prediction was that verbal reports would be biased toward meaningful fragments when Ss reported the parts that remained but not necessarily when they reported disappearances. Another purpose was to check the distributions of latencies and durations presented in Figs. 1 and 2. If Ss accurately "tracked" the filmed fragmentations, this would give additional support for the notion that the film was representative with respect to these measures.

\section{Method}

\section{Subjects}

The Ss were 52 males and 12 females.

\section{Film Preparation}

A master copy of the $\mathbf{B}$ figure was photographed and printed on high-contrast process film, resulting in an opaque. black pattern on a transparent ground. This figure was the same size as the outline Ss used in Experiment I for tracing. The simulated fragments were filmed by registering this master copy on a white copyboard and masking out with a white overlay sheet that portion which was to disappear.

The 240 tracings of fragments reported in Experiment I were arranged in a random order. and each was assigned a latency and duration using the probability distributions represented in $\mathrm{F}$ tgs.
1 and 2. The sirnulation movie was then photographed one frame at a time. During each latency period, the full figure was filmed.

Since the $240 \mathrm{fragments}$ produced a film only $13 \mathrm{~min}$ long, an additional 29 of the original fragments were sampled at random in order to make a $15-\mathrm{min}$ film. Means, confidence intervals, and standard deviations for both latencies and durations of simulated fragments are listed in Table 1 . In setting up the confidence intervals, the distributions obtained in Experiment I were treated as populations. Since the original mean latency and duration were both well within their $95 \%$ confidence intervals, the film may be considered representative of the parent population with respect to these parameters.

\section{Apparatus}

With only one important exception, the apparatus and experimental situation were essentially the same as that used in Experiment I. Instead of an electroluminescent panel at S's eye level, there was a 12-in.-sq rear-projection screen. A 35 -mm projector was mounted behind the screen so that the projected HB was 7 in. high $\times 8$ in. wide. Neutral density filters were used to reduce the luminance of the projected image to approximate that of the previous study.

\section{Procedure}

The Ss were assigned at random to the four cells of a 2 by 2 factorial design, with dimensions consisting of reporting method (verbal vs tracing) and content reported (fragments remaining vs parts that disappeared). The only constraints upon randomization in this assignment was that each group received three female $S s$ and that the cell sizes were equal.

Generally, instructions and procedure were the same as those in the previous study. The $S$ was told that at some time-during the trial the room light would come on when he touched the control switch to report a fragment. From this point on, however, instructions varied according to S's group. Those in Group DT were told to trace the part that disappeared. Those in Group RT were told to trace the parts that remained. Group DV was told to describe verbally the part that disappeared, whereas Group RV was told to describe verbally the part that remained. The Ss in both verbal groups were told that their reports would be recorded on tape and that $E$ would listen to these descriptions later. Their task, then, was to make the report as clear, complete, and unambiguous as possible so that $\mathrm{E}$ would be able to reproduce what they reported. Since it was important to have only simulated fragments uncontaminated by the real phenomenon, the instructions did not mention restriction of head, eye, or blinking movements.

The practice trials for each $S$ began where the film had stopped at the end of the previous S's expetimental trials. The point of entry into the film for experimental trials, however, was determined at random.

\section{Results and Discussion}

The proportion of meaningful fragments was calculated for each $\mathrm{S}$ by having two research assistants listen independently to the taped records of the reports of the verbal groups and trace the fragments on data sheets. These assistants also assigned the reported fragments to the meaningful or nonmeaningful sets. There were no discrepancies between the two judges for three of the four groups. but there were discrepancies in the proportion of meaningful fragments for 3 of the 16 Ss in Group DV. For these three cases. the mean of the discrepant proportions $w$ as assigned as $S^{\prime}$ 's score.

The mean proportion of meaningful fragmentations 
were .35 for Group RV, .27 for Group DV, .26 for Group RT, and .23 for Group DT. Analysis of variance failed to support the initial hypotheses. The $F$ value comparing verbal reports with tracings was 3.56 (df = $1,60 ; \mathrm{p}<.10)$. The comparison of reports of disappearances with reports of parts that remained resulted in an $F$ of $2.79(\mathrm{df}=1,60 ; p=.10)$. The $F$ for interaction was less than unity.

Two of the four groups (DT and DV) were arbitrarily selected for intensive analysis of the latencies and durations of the reported fragmentations. Since visual inspection of the frequency distributions of these two groups suggested no systematic differences, the data were pooled to form the distributions whose summary statistics are shown in Table 1. These data indicate that Ss were tracking accurately the latencies and durations of the simulated fragmentations in the film. An additional implication is that the mean values of the distributions of latencies and durations used to create the film accurately reflected those of real fragmentation phenomena.

\section{EXPERIMENT III: CONTINUOUS VERBAL REPORTS}

The outcome of Experiment II was not comparable to the effect reported by Schuck and Leahy (1966), where Ss reported disappearances verbally throughout each trial. The purpose of the present experiment was to find out if continuous verbal reporting enhances the bias toward the reporting of meaningful fragmentations.

\section{Method}

\section{Subjects}

The Ss were 15 male and 13 female students.

\section{Apparatus and Procedure}

The film, projector, and basic experimental situation were identical to those used in the previous study. The $S s$ in Group CVR ( 7 males and 7 females) were told to report verbally throughout each trial the parts of the $\mathrm{HB}$ figure that remained. The Ss in Group CVD ( 8 males and 6 females) were told to report the parts that disappeared. The portion of the instructions referring to the contact switch was omitted, since it was not used in this study.

\section{Results and Discussion}

Two judges listened independently to each S's recorded responses, traced the fragments reported, and identified these fragments as belonging to the meaningful set or not. In this way, each judge derived a proportion of meaningful fragments for the six trials of each S. Whenever discrepancies occurred, the mean of the judges' separate scores was assigned to the $\mathrm{S}$. The reliability of the judges was high $(r=.92)$. The mean proportion for Group CVR was .54 as compared to .26 for Group CVD. These means were significantly different, as indicated by a " $t$ " of $6.60(p<.001)$, thus supporting the hypothesis that continuous verbal reporting markedly increases response bias

\section{EXPERIMENT IV: \\ REPORTING ACCURACY AS A FUNCTION OF THE COMPLEXITY OF THE FRAGMENT}

The film used in the previous two experiments did not provide a way of checking upon the accuracy of a specific report, since the projector could not be stopped quickly enough to be sure which fragment was the last one $\mathrm{S}$ saw.

In order to eliminate this difficulty, an electroluminescent alphanumeric panel was used in this experiment. The fragmentation phenomenon was simulated by programming specific line segments to disappear and reappear in keeping with the distributions of latencies and durations observed in Experiment $\mathrm{I}$. Consequently, the specific fragment to be reported at the end of each simulation trial was known. Since it is most unlikely that Ss would have any difficulty reporting highly familiar fragments (such as common letters, numerals, or symbols) only unfamiliar fragments were simulated, and the complexity of these fragments. as defined by the number of line segments remaining, was varied. The major purpose of this project, then, was to study the accuracy of tracings of unfamiliar simulated fragments as a function of their complexity.

\section{Method}

\section{Subjects}

The Ss were 8 students, 5 of whom were paid for their participation.

\section{Apparatus}

The alphanumeric panel, $4 \mathrm{in}$. high by $3 \frac{112}{\mathrm{in}}$. wide, was mounted at S's eye level. A Kennedy Model trp- 62 magnetic tape programmer was used to program presentation of the 14 segments illustrated in Fig. 4 .

\section{Procedure}

The $\mathrm{S}$ was shown simulated fragmentations for several minutes and then read these instructions: "As you just noticed, a pattern like this appears on the screen and changes from moment to moment. I am interested in finding out how well you can report these changes. At some time during the trial, the screen will go blank. When this happens, immediately trace on the data sheet the last pattern you saw by tracing the parts that remained. Please make your tracing as true a picture as you can of that last pattern before the screen went blank. There will be $30 \mathrm{sec}$ between trials. Are there any questions?"

Each data sheet contained a faint, light blue outline of the 14 segments of the alphanumeric display. The $S$ received four sessions of 18 trials each. Two sets of seven segments were selected at random to constitute the intact figure or pattern for two of S's experimental sessions. On the remaining two sessions. $S$ received the complementary sets as the intact pattern. One restriction on the random selection of these sets was that no set 


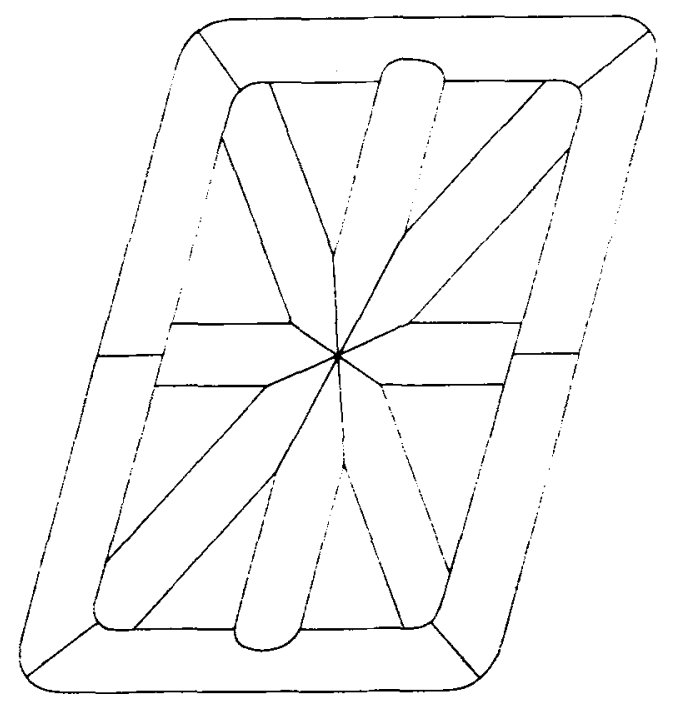

Fig. 4. A schematic diagram of the 14 "line segments" making up the alphanumeric panel.

or its complement was permitted to form a letter, numeral, or common symbol.

Throughout each trial, the number of segments disappearing was selected at random, but the complexity of the last fragment of each trial was experimentally manipulated and varied so that from one to six segments remained. Also, there were exactly three examples of each of these six levels of complexity in each 18 -trial session.

\section{Results and Discussion}

An error was defined as either failing to trace a segment that actually remained or tracing a segment that did not remain. The proportion of tracings containing at least one error was then calculated for each of the six levels of complexity for each S. Fig. 5 shows the resulting mean proportions of errors and their standard deviations.

No $S$ made an error on any trial when only one segment remained. but mean errors increased as the number of segments remaining increased. Eighty-four percent of the errors involved confusion among the seven elements of the initial, intact pattern. For these kinds of fragments. $S$ probably should not be required to report the disappearance of more than one or two elements.

\section{EXPERIMENT V: \\ THE FRAGMENTATION OF LUMINOUS LINE SEGMENTS AS A FUNCTION OF LENGTH, WIDTH, AND ORIENTATION}

Craig and Lichtenstein (1953) and Goldstein (1967) found that the frequency with which a stimulus line disappears is a function of the orientation of the line. Other evidence indicates that the retinal position of a stimulus is an important factor affecting the frequency of its disappearance (Clarke, 1957; Ronchi \& Conticelli, 1961).

In view of this kind of evidence, factors other than the complexity must be considered in designing a stimulus pattern for the assessment of Hebb's hypothesis. This project focused upon several of these. Specifically, the purpose was to study the effect and possible interaction of length, width, and angular orientation on the disappearance of a luminous line segment or rectangle.

\section{Method}

\section{Subjects}

The Ss were 15 females and 21 males.

\section{Apparatus and Procedure}

The $S$ sat in a darkened room and viewed a luminous line segment radiating in his picture plane either vertically (360 deg), diagonally $(315 \mathrm{deg})$, or horizontally $(270 \mathrm{deg})$ from a point of origin at eye level just $50 \mathrm{in}$. in front of him. The luminous line was created by masking the 12-in.-sq electroluminescent lamp and mounting it on a large circular disk of $3 / 4$-in. plywood. The plywood disk was $4 \mathrm{ft}$ in diam, was painted flat black, and was mounted on an axis so that $E$ could change the orientation of the luminous line by rotating the disk to the desired angle. The $E$ could adjust two vernier gauges that were independently connected to edges of the mask, one determining the width, the other the length of the line segment or rectangle. Voltage input to the lamp was adjusted so that the luminance was approximately . $1 \mathrm{fL}$.

Seven males and five females were assigned at random to each of three groups. All $\mathrm{Ss}$ in each group received nine experimental trials corresponding to the factorial combinations of three lengths (corresponding to visual angles of 3.6, and $12 \mathrm{deg}$ ) and the three orientations of vertical, diagonal, and horizontal. The

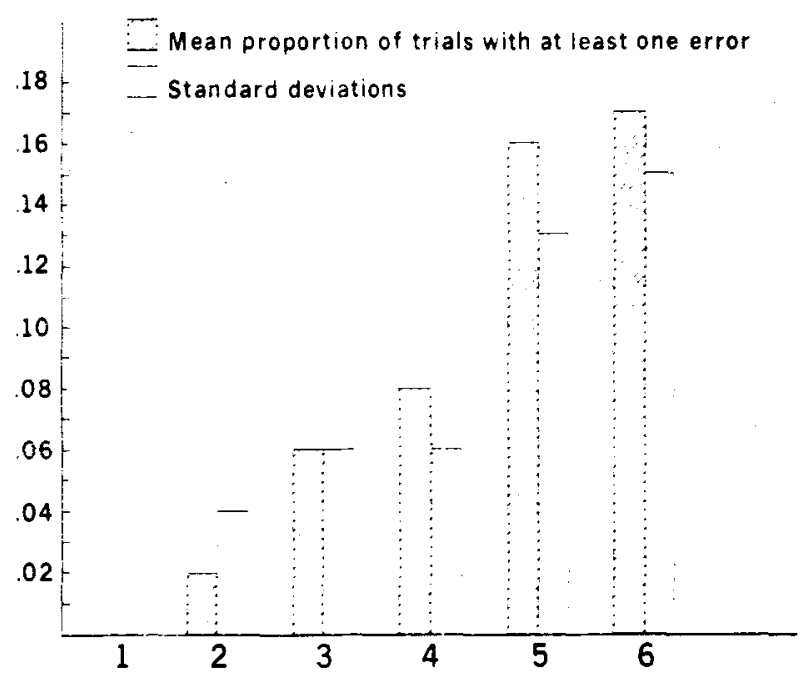

Number of Elements in Fragment.

Fig. 5. Means and standard deviations of the proportion of incorrect trials in Experiment IV. 
order in which these treatments were presented was randomized for each S.

The Ss in one group viewed a wide segment. those in the second group viewed a segment of medium width, and those in the third group viewed a narrow segment. These widths corresponded to visual angles of 104,52 , and $26 \mathrm{~min}$.

The $S$ reported the disappearance of part or all of the line segment by pressing a button-type of handswitch connected to an electromagnetic counter. Head movements were restrained by use of a headrest and bite board. Monocular vision was insured by having $S$ wear lensless spectacle frames in which the nonpreferred eye was occluded.

Each $S$ received from three to six practice trials before the experimental session began. Practice trials consisted of a random sequence of the treatment conditions and were discontinued when $S$ reached the criterion of two successive trials in which he reported at least one fragmentation. Both practice and experimental trials began with the room lights going out. Five seconds later, the luminous line segment appeared and lasted for $40 \mathrm{sec}$. The intertrial interval was $30 \mathrm{sec}$.

\section{Results}

The mean numbers of reported disappearances were $3.78,4.69$, and 5.22 for the short, medium, and long lengths, respectively, and the $F$ ratio for this effect was $19.155(\mathrm{p}<.001, \mathrm{df}=2,66)$. For orientation, the mean values were $4.53,5.14$, and 4.02 for vertical, diagonal, and horizontal line segments, respectively. The corresponding $F$ value was $9.825(\mathrm{p}<.001$, $\mathrm{df}=2,66)$. Neither the $F$ ratios for width nor those for the interaction terms approached significance.

\section{EXPERIMENT VI: THE WIDTH EXPERIMENT} 4

There are two possible explanations for the failure in the previous study to find a significant effect due to width. One is that width does not affect disappearances. Another is that the width of a luminous line segment or rectangle really does affect disappearances, but that the statistical design was insensitive to that particular treatment. Width was a between-Ss condition, whereas both orientation and length involved within-S comparisons. In order to evaluate the possibility of this kind of error, another study was conducted in which width was a within-S variable.

\section{Method}

\section{Subjects}

Eleven males and 2 females from introductory psychology classes served as Ss. All received extra credit for volunteering.

\section{Procedure}

The basic apparatus was the same as that used in the previous study. The length of the luminous line was held constant, however, at $3 \mathrm{deg}$ and the orientation was always vertical. The widths were varied and included visual angles of $33,52,71,90$, and $109 \mathrm{~min}$.

Each $S$ received 5 practice trials, one with each of the five different widths, in a random order. These were followed by 10 experimental trials. consisting of 2 trials for each of the five widths, also in a random order. In all other respects, the method and procedure were identical to the previous study.

\section{Results}

The mean number of disappearances for the five different widths. from narrow to wide, were 4.62,5.19, $4.12,4.88$, and 4.27 . The $F$ ratio of 1.43 was not significant $(\mathrm{df}=4,48)$. Thus, within the range sampled here, width was not an important factor influencing the frequency of disappearances.

\section{EXPERIMENT VII: AN EXACT TEST OF HEBB'S HYPOTHESIS CONCERNING MEANINGFUL DISAPPEARANCES}

Two major criteria must be considered for the proper design of a study to test Hebb's prediction that disappearances will result in a preponderance of meaningful fragmentations. One is that the reporting task must be well within S's capability. Although this seems trite when stated, it is a criterion which apparently has not often been met in the study of fragmenting images. Secondly, the stimulus pattern should be one in which differences in the frequency of meaningful and nonmeaningful fragmentation cannot be attributed to contaminating variables.

For the present study, an I-shaped pattern was selected on the basis of these criteria. The vertical and horizontal lines of this pattern were in the proper proportion so that when the bottom horizontal line disappeared, the part that remained looked like an uppercase letter $T$. When the top horizontal line disappeared, the remaining figure looked like an inverted $T$. The purpose of the first part of the present study was to provide empirical validation for the notion that an upright $T$ is a more meaningful form than an inverted $T$. In the second part, Ss viewed a luminous I-shaped pattern in a darkened room and reported only those disappearances resulting in either a $\mathrm{T}$ or an inverted $\mathrm{T}$. The proportion of $\mathrm{T}$ reports was observed while manipulating the size of the I pattern and the location of the fixation point.

\section{Part I}

\section{Method}

Subjects. The Ss were 252 volunteers, about half male and half female.

Apparatus. The stimulus patterns were drawn in india ink on $8 \times 10$ in. pieces of white construction paper and projected on a screen by the manual operation of an opaque projector. The $E$ timed the stimulus presentations and the interstimulus intervals by visually monitoring a continuously cycling Gray- $\mathrm{Lab}$ timer.

Procedure. The Ss were gathered in four groups of from 61 to 67 students each in a classroom especially designed for 
audio-visual purposes. The Ss were told that the study was one phase of a research project concerned with the meaningfulness of visual patterns and that this particular project was especially concerned with how many mental associations they could make to various kinds of simple patterns. An example was given of the kinds of associations a typical S might make to the outline of a star. Since not enough time was available for their listing specific associations, Ss estimated the number of associations each projected pattern might bring to mind, using the following scale: $A$. very many: $B$, above average; $C$, average; $D$, below average; and $E$, none. Each pattern was presented for $3 \mathrm{sec}$, with $5 \mathrm{sec}$ between presentations. The Ss recorded their answers on IBM answer sheets. Each group received a different random ordering of the patterns.

Results

The ratings from $E$ to $A$ were converted to numerical scores ranging from -2 for the $E$ rating to +2 for the $A$ rating. Although data were gathered for some 150 different patterns, only those pertaining to $T$, inverted $\mathrm{T}$, and several comparison patterns will be considered here.

The mean rating for $\mathrm{T}$ was 1.15 with a $95 \%$ confidence interval ranging from 1.02 to 1.28 . This was quite comparable to the letters and symbols from the set used in Project II to operationally define the meaningful fragmentations of the $\mathrm{HB}$ figure. For example, mean ratings were 1.14 for $\mathrm{H}, 1.09$ for $\mathrm{h}$, and 1.37 for + . The mean rating was .71 for the inverted $\mathrm{T}$, with a confidence interval from .57 to .85 .

As measured by rated association value, the meaningfulness of the $T$ figure is comparable to other common letters and symbols and significantly more meaningful than its upside down counterpart.

\section{Part II}

\section{Method}

Subjects. The Ss were 42 females and 30 males.

Apparatus and procedure. The $\mathrm{S}$ sat at a table 42 in. in front of a flat-black plywood partition. An electroluminescent panel, 12 in. square, was mounted on the partition and centered at S's eye level. The panel was overlayed with one of three photographic negatives of an I-shaped figure on high-contrast process film. The large figure was $6 \frac{3 / 4}{\mathrm{in}}$. high $\times 5 \frac{1}{4} \mathrm{in}$. wide and was made up of lines $1 / 2$ in. wide. The medium-sized figure was $41 / \mathrm{in}$. high and the small figure was $2 \frac{1 / 2}{\mathrm{in}}$. high. The visual angles subtended were approximately 9.6 . and $3 \mathrm{deg}$ for large. medium. and small figures. respectively. The other dimensions for the medium and small figures were proportional to those of the large pattern.

On the table in front of S were two response keys. The $S$ was told to press one when a disappearance left a recognizable $T$ intact and to press the other when a recognizable. intact upside-down $T$ remained. Half of the $S s$ in each treatment group used the right key to report $T$ fragmentations: the other half used the left key for this report. In order to insure monocular viewing. each $\mathrm{S}$ wore spectacle frames which occluded the nonpreferred eve. A headrest and bite bar helped $S$ maintain stabilization.

One group was told to fixate the I pattern at the intersection of the vertical bar and the upper horizontal line. Another was told to fixate at the bottom intersection. A third was told to fixate at the midpoint of the vertical line, that is, the point equidistant from the two horizontal lines. Since it was possible that fixating the midpoint of the vertical line was a more difficult task than fixating either of the two intersections, a control group was added. These $S s$ were provided with a small fixation dot just $3 / 16$ in. in diam and equidistant from the top and bottom horizontal lines of the figure. For half of the controls, this fixation point was adjacent to the right edge of the vertical line segment. For the remaining half, it was adjacent to the left edge. Each $S$ received 3 practice trials followed by 12 experimental trials. Except for the differences mentioned here, the apparatus, method, and procedure were identical to those used in Experiment $\mathrm{I}$.

The experimental design was a factorial combination of the three sizes of patterns and the four fixation conditions, with six Ss assigned to each of the 12 cells.

\section{Results and Discussion}

The number of $T$ and inverted- $T$ fragments was recorded and used to calculate the proportion of $T s$ reported by each $S$ over his 12 trials. The subsequent analysis was performed on arc sine transformations of these proportions. The means of the transformed scores were $70.63,48.45,43.85$, and 25.10 for those fixating at the top, midline, middle dot, and bottom of the I figure, respectively, and an $F$ ratio of 43.00 indicated significant differences among these means $(\mathrm{df}=3,60$; $p<.01)$. The estimated omega squared for this effect was .66 , revealing that the locus of fixation accounted for an estimated $66 \%$ of the variance. Both of the other $F$ values, one for the effect of size and the other for the interaction of size with fixation, were less than unity.

A Scheffé post hoc test indicated no significant difference between the two middle fixation conditions, since the $F$ ratio for this contrast was also less than unity. The pooled mean for the midline and the middot fixations was 46.87 , with a $95 \%$ confidence interval ranging from 41.75 to 52.00 . Scheffé tests did indicate that the other two fixation conditions were significantly different from the average of the two middle-fixation conditions, however. The $\mathrm{F}$ ratio corresponding to the contrast of the top fixation with the average of the two middle-fixation conditions was 31.56. The corresponding $F$ value for the contrast of the bottom fixation with the average of the middle conditions was 23.34. Both ratios exceeded 12.39 , the adjusted critical value of $F$ for an alpha level of $.01(\mathrm{df}=3,60)$.

In this study. possible contaminating effects due to visual angle, foveal position, or angular orientation were hopefully eliminated by the symmetry of the original pattern and by the identical characteristics (aside from rotation) of the $T$ and inverted $T$ fragments. The results. however, failed to support Hebb's concept of the relative stability of meaningful as compared to less meaningful forms. Distance from the locus of fixation, rather than meaningfulness. accounted for the major portion of the variance. 


\section{GENERAL CONCLUSIONS}

The series of studies reported here call attention to several important methodological strictures for those researchers interested in disappearance phenomena. In general, discrete verbal reports and tracings appear adequate for representing only the very simplest of fragments, those involving no more than one or two perceptual elements (such as line segments). Continuous verbal reporting accentuates the bias toward meaningful fragments. Moreover, locus of fixation is a potent factor affecting the reporting of disappearances. Key or button pressing, however, was found to be a satisfactory way to measure the frequency, latency, or duration of disappearances. No support was found for Hebb's hypothesis.

Although reporting the fragmentation of an $\mathbf{B}$ figure seems sufficiently simple and straightforward, the present data lead to an entirely different conclusion; they suggest that we are only beginning to realize how complex this kind of experimental situation really is.

\section{REFERENCES}

Clarke, F. J. J. Rapid light adaptation of localized areas of the extra-foveal retina. Optica Acta, 1957, 4, 69-77.
Clarke, F. J. J. A study of Troxler's effect. Optica Acta, 1960.7. 219-236.

Clarke, F. J. J. Visual recovery following local adaptation of the peripheral retina. Optica Acta, 1961, 8, 121-135.

Craig, E. A., \& Lichtenstein, M. Visibility-invisibility cycles as a function of stimulus orientation. American Journal of Psychology, 1953, 66, 554-563.

Forde, J., Piggins, D., \& MacKinnon, G. E. Fragmentation and regeneration of retinal images. Paper presented at the meeting of the Canadian Psychological Association, June 1966.

Gamer, W. R. Uncertainty and structure as psychological concepts. New York: Wiley, 1962.

Goldstein, A. G. Retinal rivalry and Troxler's effect. Psychonomic Science, 1967, 7, 427-428.

Hebb, D. O. The semiautonomous process: Its nature and nurture. American Psychologist, 1963, 18, 16-27.

McKinney, J. P. Disappearance of luminous designs. Science, $1963,140,403-404$.

Pritchard, R. M., Heron, W., \& Hebb, D. O. Visual perception approached by the method of stabilized images. Canadian Journal of Psychology, 1960, 14, 67-77.

Ronchi, L., \& Conticelli, M. The "fading out" of a fixated, test-field. Monocular and binocular experiments. Atti Fondazione Giorgio Ronchi, 1961, 16, 643-653.

Schuck, J. R., \& Leahy, W. R. A comparison of verbal and nonverbal reports of fragmenting visual images. Perception \& Psychophysics, 1966, 1, 191-192.

(Received for publication June 15, 1971; revision received October 30,1972 .) 